\title{
Miller Code Usage in Visible Light Communications under the PHY I Layer of the IEEE 802.15.7 Standard
}

\author{
Alin-Mihai Cailean ${ }^{1,2}$, Barthélemy Cagneau ${ }^{1, *}$, Luc Chassagne ${ }^{1}$, Mihai Dimian ${ }^{2}$, and Valentin Popa ${ }^{2}$ \\ ${ }^{1}$ Université de Versailles, Laboratoire d'ingénierie des systèmes de Versailles, Vélizy-Villacoublay, France \\ ${ }^{2}$ Stefan cel Mare University, Department of Computers Electronics and Automation, Suceava, Romania \\ *Corresponding author (E-mail : barthelemy.cagneau@uvsq.fr)
}

\begin{abstract}
This paper approaches the issues concerning the usage of the delay modulation as a coding technique used for outdoor Visible Light Communications (VLC) under PHY I layer of the IEEE 802.15.7 standard. We perform a comparative analysis between the Manchester code, as a traditional code, specified by the upper mentioned standard and the Miller code as a possible candidate for outdoor MIMO applications. Simulation and experimental results are provided, offering an overview over the multi-channel, flickering and Bit Error Ratio (BER) performances.
\end{abstract}

Key words - visible light communications; light emitting diode; intensity modulation; delay modulation; Manchester coding; 802.15.7.

\section{INTRODUCTION}

LED systems began to be used in several applications because of specific advantages. Besides lighting, LEDs can enable VLC. VLC is safe for the human health unlike radiofrequencies waves which are considered as a cause of cancer in humans [1] or like infrared communications which can cause thermal damage on the cornea. VLC also offers worldwide unregulated unlimited bandwidth, having the potential for extremely high data rates that can go above 1 Gbps [2].

One particular field of applications for VLC is the Intelligent Transportation System (ITS). VLC allows for Infrastructureto-Vehicle (I2V) and for Vehicle-to-Vehicle (V2V) communications (see [3], [4] and [5], [6]). Enabling inter-vehicle communication may substantially improve the safety and the efficiency of the transportation system, addressing up to $81 \%$ of all vehicle crashes [7].

In the recent years, LED-based lighting has begun to be integrated in the transportation system. The car manufactures began to replace the halogen lamps by LEDs, whereas city authorities use LEDs systems to replace the classical street lighting systems and integrate them in traffic lights. This enables VLC to be an ubiquitous technology capable of a high market penetration, contributing to the success of the ITS.

This paper presents an analysis of the Miller coding technique and of its appropriateness for VLC outdoor usage in ITS application. Simulation results show that in terms of bandwidth and channel coexistence, the Miller code clearly outperforms the Manchester code. Experimental results confirm that in terms of BER, both codes exhibit same performances. Since the IEEE 802.15.7 [8] standard choses the usage of the Manchester code taking into consideration its flickering performances, the paper also analyses the flickering performances of the Miller code. As far as we know, this is the first detailed analysis that focuses on the Miller code for VLC usage.

\section{Modulation techniques Used IN VLC}

Intensity Modulation (IM) is usually considered to be the most appropriate modulation technique for VLC. IM implies to modulate the desired waveform onto the instantaneous power of the carrier. The receiver extracts the data from the modulated light beam by using Direct Detection (DD). The photodetector generates a current proportional to the incident power. This current is thus transformed into a voltage by a transimpedance circuit and then the signal is processed through several filters and amplification stages until the data signal is reconstructed. For short, this is also the working principle of the system we have developed and that is detailed in section IV.

Depending on the application, many modulations techniques were proposed and investigated for VLC usage. Orthogonal Frequency Division Multiplexing (OFDM) [9] and discrete multi-tone modulations (DMT) [10] techniques offer the premises for high data rates and are mainly used for indoor static applications. However, complex modulations may lead to complex transceivers. For applications that require dimming, Pulse Width Modulation (PWM) [11] is considered as an alternative. For low data rates applications meant for outdoor usage, where the Signal-to-Noise Ratio (SNR) is low, simpler modulations techniques are generally used. On-OffKeying (OOK) is a solution quite efficient. OOK modulation is regularly used with Not Return to Zero (NRZ) or with Manchester coding. The use of Pulse Position Modulation (PPM) or of Inverted-PPM [12] have also been investigated. Compared with OOK, PPM and I-PPM can achieve higher data rates but require more bandwidth, higher peak power and are more sensitive to noise. In order to reduce the noise effect, the use of Direct Sequence Spread Spectrum (DSSS) sequence inverse keying (SIK) has been investigated and implemented [3]. This type of coding has error detecting capabilities and enables multiple transmitters.

The IEEE 802.15.7 standard for wireless optical communications using visible light defines for the PHY I outdoor 
usage, the utilization of OOK and of Variable Pulse Position Modulation (VPPM) as possible modulation techniques. VPPM is an improved modulation technique that combines the characteristics of pulse position modulation (2-PPM) for nonflicker and of pulse-width modulation (PWM) for dimming control and brightness control. VPPM is similar to 2-PPM but allows the pulse width to be controlled for light dimming. All VPPM PHY I modes use 4B6B encoding. VPPM is mostly intended for applications that require dimming. For OOK, the standard mentions the usage of the Manchester code with five different data rates: 11.67, 24.44, 48.89, 73.3 and $100 \mathrm{~kb} / \mathrm{s}$.

\section{Simulation RESUlts}

A. Considerations on multi-channel capabilities for Manchester and Miller codes

The Manchester code, also known as the biphase code, is a classical code, in which ' 0 ' is encoded as ' 01 ' and ' 1 ' becomes '10'. The main advantages of this code are DC balance, easy clock and data recovery, decent BER performances. However, even if it has plenty of advantages, Manchester code requires high bandwidth compared to other common codes. For example, it requires twice the bandwidth of Not-Return to Zero (NRZ). On the other hand, the Miller code [13], also known as delay modulation, appears to be more convenient for Multiple Input Multiple Output (MIMO) applications, since it uses the bandwidth more efficiently. The Miller code can be easily constructed using the Manchester code. In Miller code, a ' 1 ' is encoded as a transition on the mid-bit position, a ' 0 ' following a ' 1 ' is encoded as no transition on the entire bit period, whereas a '0' following a ' 0 ' is encoded as a transition on the beginning of the second bit period. The Miller code has very good timing content, and carrier tracking is easier than Manchester coding.

The Power Spectral Densities (PSD) for these three codes are given by 1,2 and 3 .

$$
\begin{aligned}
& S_{N R Z}(f)=\frac{V^{2} T}{4}\left(\frac{\sin (\pi f T)}{\pi f T}\right)^{2}+\frac{V^{2}}{4} \delta(f) \\
& S_{M a n}(f)=V^{2} T\left(\frac{\sin (\pi f T / 2)}{\pi f T / 2}\right)^{2} \\
& S_{M i l}(f)= \frac{V^{2} T}{2(\pi f T)^{2}(17+8 \cos (2 \pi f T)} \\
& \times {[23-2 \cos (\pi f T)-22 \cos (2 \pi f T)} \\
&-12 \cos (3 \pi f T)+5 \cos (4 \pi f T) \\
&+12 \cos (5 \pi f T)+2 \cos (6 \pi f T) \\
&-8 \cos (7 \pi f T)+2 \cos (8 \pi f T)]
\end{aligned}
$$

where $V$ is the signal amplitude and $T$ the modulation period. $f$ is the frequency for which the PSD is calculated.

Even if the performances of the NRZ code are not addressed by this paper, we introduce it as a reference. The corresponding curves for a modulation frequency of $11.67 \mathrm{kHz}$ are plotted in figure 1 .

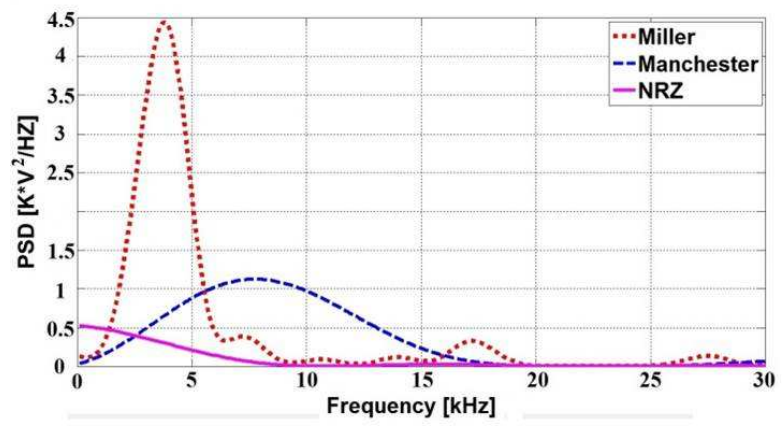

Figure 1. PSD for NRZ, Manchester and Miller code at $11.67 \mathrm{kHz}$.

It can be noticed that the Manchester code requires twice the bandwidth of the NRZ code. For the Miller code's PSD, the maximum energy is reached for a frequency around $2 / 5$ of the modulation frequency.

Figures 2 and 3 illustrate the coexistence of five adjacent channels for the data rates specified by the 802.11.7 standard for OOK, Manchester and Miller codes respectively.

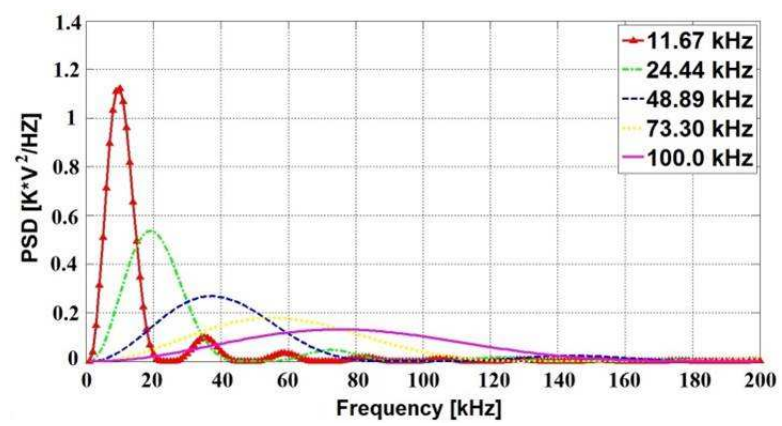

Figure 2. Simulation for five channels configuration, using the Manchester code.

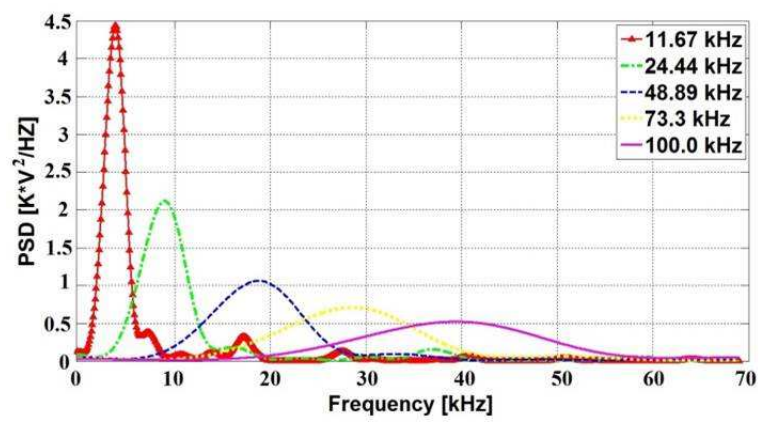

Figure 3. Simulation for five channels configuration, using the Miller code.

It can be seen that for Manchester code, the five carriers overlap, making the separation quite difficult and introducing decoding errors. Regarding the Miller code, the five channels can be well distinguished. This allows for the five sub-carriers to be more easily processed by bandpass filters, either analog or digital. 


\section{B. Flickering issues concerning the Manchester and the Miller code}

The VLC technology adds communication capabilities to the classical lighting. However, VLC must not affect in either way the primary role of the appliance, which is lighting or signaling. Flickering mitigation is one of the main concerns regarding the VLC. Flickering represents the light intensity fluctuation caused by the modulation technique. It is classified as inter-frame flickering and as intra-frame flickering. Flickering is prevented when the light intensity changes within the Maximum Flickering Time Period (MFTP). In this case the human eye does not notice the light intensity changes. Even if an optimal flicker frequency is not widely accepted, it is considered that a MFTP smaller than $5 \mathrm{~ms}(200 \mathrm{~Hz})$ is safe [8]. The IEEE 802.15.7 standard specifies the use of Run Length Limiting (RLL) line coding as a technique for preventing perceivable flickering. Manchester, 4B6B or 8B10B codes are some examples. The RLL codes prevent long runs of $1 \mathrm{~s}$ and $0 \mathrm{~s}$ that can cause flickering and also ensure better clock and data recovery. For outdoor usage, the IEEE 802.15.7 standard specifies for the OOK, the usage of Manchester as a technique for preventing perceivable flickering, whereas for VPPM, it specifies the usage of the 4B6B code. For both modulations, the non-flickering characteristic is achieved by having the same brightness for bits ' 1 ' and ' 0 '.

Due to its characteristics, the Miller code cannot ensure the same brightness for bits ' 1 ' and '0'. For bit '1', every bit has the same brightness. But, for ' 0 ', the brightness can be either twice the brightness of '1' or it can be zero. Under these considerations, instead of determining the brightness of Miller coded messages on an individual bit level, we determine it on a byte level. It seems that as long as the modulation period is at least eight of the MFTP, if each byte's brightness is equal, no noticeable flickering is induced.

To determine the flickering characteristic of the Miller code, we have performed several simulations. A number of $10^{5}$ messages, containing 64 random ASCI characters (512 bits) were generated. The messages were encoded using the Miller code. The brightness of each byte is determined by measuring the 'lights on' time as a percentage of the total byte time. We consider that $100 \%$ brightness is achieved when the light is on for half of the byte time (as for the Manchester code).

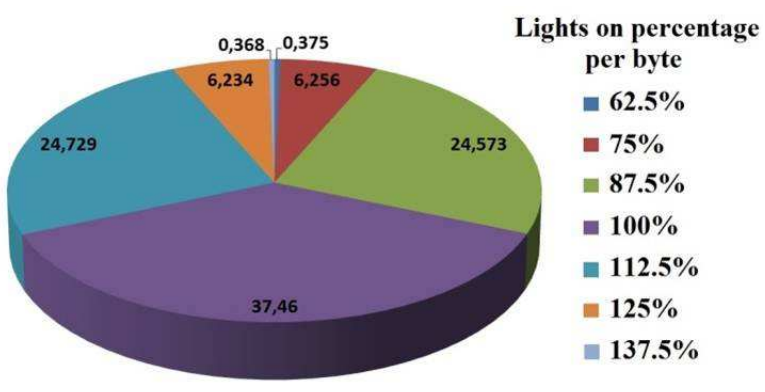

Figure 4. Simulation results showing the bytes percentage for different brightness intensities.

The figure 4 shows that the brightness of the bytes is $100 \%$ for $37.46 \%$ of the cases, varies in $49 \%$ of the cases by $\pm 12.5 \%$, in $12.5 \%$ of the cases by $\pm 25 \%$, whereas in $0.7 \%$ of the cases by $\pm 37.5 \%$. Regarding these results, we can conclude that unlike the Manchester code, the Miller code exhibits some brightness variations from one byte to another. However, since the byte period is significantly shorter than the MFTP, flickering at the byte level cannot be perceived.

In figure 5, we determine the brightness of each MFTP, for the five data rates mentioned by the standard.

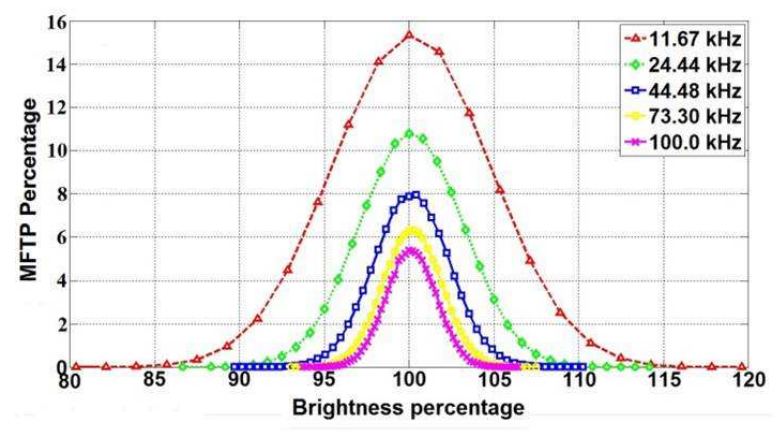

Figure 5. Simulation results showing the percentage of MFTP for different brightness percentages.

As showed in figure 5, the MFTP's brightness is a Gaussian distribution, centered on the $100 \%$ brightness intensity, which gets narrower as the modulation frequency increases. The results show that even at the lowest data rate, more than $96 \%$ of the MFTPs have an oscillation bellow $\pm 10 \%$. Furthermore, the human eye does not have a linear response to changes in light intensity. According to [14], the relation between the perceived light and the measured light is given by 4 .

$$
\text { Perceived light }(\%)=100 \times \sqrt{\frac{\text { Measured light }(\%)}{100}}
$$

This relation reduces brightness variation sensation, limiting the flickering effect perceived by the human eye.

\section{HARDWARE IMPLEMENTATION AND EXPERIMENTAL RESULTS}

For the final tests, we determine the BER performances of the two codes. These tests are performed using a VLC communication system that we have developed (see figure 6). The system is meant to be used for traffic safety information. It broadcasts data between a traffic light based on LED to a vehicle (information about the color of the traffic light and the countdown before the next color change). The emitter was developed based on a commercial LED traffic light on which we have added a controller unit that performs data encoding and LEDs switching. The receiver consists of a photodiodebased light detection module, several filtering and amplification stages and a signal processing. Information treatment and decisions taking unit are performed with a low-cost 8-bit microcontroller. In our prototype, the microprocessor can be switched either on the Manchester or Miller code in order to test different configurations. 


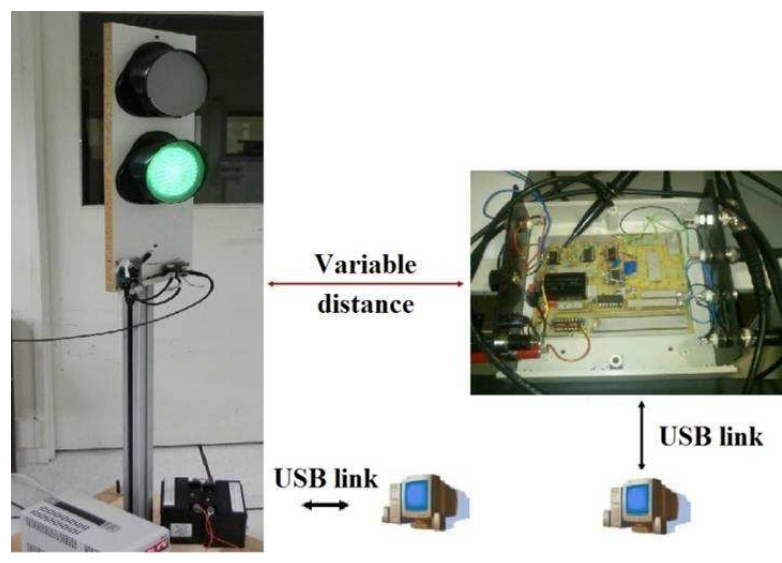

Figure 6. Visible light communications system consisting of a traffic light (red or green) emitter and a receiver.

Table I

BIT ERROR RATIO PERFORMANCES AT $15 \mathrm{KHz}$

\begin{tabular}{|c|c|c|c|}
\hline Data Coding & $\begin{array}{c}\text { Emitter receiver } \\
\text { distance }\end{array}$ & BER & $\begin{array}{c}\text { Testing } \\
\text { conditions }\end{array}$ \\
\hline Manchester & \multirow{2}{*}{$1-50 \mathrm{~m}$} & $10^{-7}$ & \multirow{2}{*}{$\begin{array}{c}\text { Outdoor with } \\
\text { daylight }\end{array}$} \\
\hline Miller & & $10^{-7}$ & \\
\hline Manchester & \multirow{2}{*}{$1-20 \mathrm{~m}$} & $10^{-7}$ & \multirow{2}{*}{$\begin{array}{l}\text { Indoor with } \\
\text { neon lights }\end{array}$} \\
\hline Miller & & $10^{-7}$ & \\
\hline
\end{tabular}

Tests were performed under various conditions, to determine the BER for the two codes. The data transmission was made at a $15 \mathrm{kHz}$ modulation frequency.

The results are detailed in [15] and summarized in table I. We demonstrate that both codes exhibit the same BER performances, at least at the $10^{-7}$ level. The developed system is able to maintain the BER lower than $10^{-7}$ for distances that increase up to 50 meters and in different testing conditions for both codes. Even in the presence of light perturbations, represented by moderate sun or by indoor neon lights, the BER performances are the same for the two codes. We mention that no error correction techniques were used for these experiments.

\section{CONCLUSION}

This paper presents a comparative analysis over the coding techniques used in VLC, focusing on the Manchester and on the Miller codes. The results show that in terms of BER, up to the $10^{-7}$ level, Manchester and Miller code have similar results, which was experimentally verified. However, in terms of spectral distribution, Miller code clearly outperforms Manchester code offering the premises for MIMO applications. Since the IEEE 802.15.7 standard, choses the usage of the Manchester, taking into account its flickering performances, the paper also analyses the flickering performances of the Miller code. The results show that even at modulation frequencies as low as $11.67 \mathrm{kHz}$, the flickering effect is very limited. However, the effects of this limited flickering must be further investigated to determine if there is any negative effect on the human health.

\section{ACKNOWLEDGMENTS}

This work was supported by the competitiveness cluster in automobile and public transport Mov'eo and the Co-Drive project.

\section{REFERENCES}

[1] W. H. Organization, "Fact sheet 193," Electromagnetic fields and public health: mobile phones, June 2011.

[2] S. Zhang, S. Watson, J. McKendry, D. Massoubre, A. Cogman, E. Gu, R. Henderson, A. Kelly, and M. Dawson, "1.5 gbit/s multi-channel visible light communications using cmos-controlled gan-based leds," Lightwave Technology, Journal of, vol. 31, no. 8, pp. 1211-1216, April 2013.

[3] N. Kumar, N. Lourenco, D. Terra, L. Alves, and R. L. Aguiar, "Visible light communications in intelligent transportation systems," in Intelligent Vehicles Symposium (IV), 2012 IEEE, June 2012, pp. 748-753.

[4] M. Akanegawa, Y. Tanaka, and M. Nakagawa, "Basic study on traffic information system using led traffic lights," Intelligent Transportation Systems, IEEE Transactions on, vol. 2, no. 4, pp. 197-203, Dec 2001.

[5] A. Cailean, B. Cagneau, L. Chassagne, S. Topsu, Y. Alayli, and J.-M. Blosseville, "Visible light communications: Application to cooperation between vehicles and road infrastructures," in Intelligent Vehicles Symposium (IV), 2012 IEEE, June 2012, pp. 1055-1059.

[6] I. Takai, S. Ito, K. Yasutomi, K. Kagawa, M. Andoh, and S. Kawahito, "Led and cmos image sensor based optical wireless communication system for automotive applications," Photonics Journal, IEEE, vol. 5, no. 5, pp. 6801418-6801418, Oct 2013.

[7] U. D. of Transportation Research and I. T. Administration, "Report: Frequency of target crashes for intellidrive safety systems," October 2010.

[8] "Ieee draft standard for information technology-telecommunications and information exchange between systems-local and metropolitan area networks-specific requirements-part 15.7: Standard for short-range wireless optical communication using visible light," IEEE P802.15.7/D8, April 2011, pp. 1-306, May 2011.

[9] H. Elgala, R. Mesleh, H. Haas, and B. Pricope, "Ofdm visible light wireless communication based on white leds," in Vehicular Technology Conference, 2007. VTC2007-Spring. IEEE 65th, April 2007, pp. 21852189.

[10] J. Vucic, C. Kottke, S. Nerreter, K.-D. Langer, and J. Walewski, "513 mbit/s visible light communications link based on dmt-modulation of a white led," Lightwave Technology, Journal of, vol. 28, no. 24, pp. 3512-3518, Dec 2010.

[11] H. Sugiyama, S. Haruyama, and M. Nakagawa, "Brightness control methods for illumination and visible-light communication systems," in Wireless and Mobile Communications, 2007. ICWMC '07. Third International Conference on, March 2007, pp. 78-78.

[12] J.-H. Yoo, R. Lee, J.-K. Oh, H.-W. Seo, J.-Y. Kim, H.-C. Kim, and S.Y. Jung, "Demonstration of vehicular visible light communication based on led headlamp," in Ubiquitous and Future Networks (ICUFN), 2013 Fifth International Conference on, July 2013, pp. 465-467.

[13] M. Hecht and A. Guida, "Delay modulation," Proceedings of the IEEE, vol. 57, no. 7, pp. 1314-1316, July 1969.

[14] M. Rea, "Lighting handbook," Illumination Engineering Society of North America (IESNA), p. 9th ed., July 2000.

[15] A. Cailean, B. Cagneau, L. Chassagne, S. Topsu, Y. Alayli, and M. Dimian, "A robust system for visible light communication," in Wireless Vehicular Communications (WiVeC), 2013 IEEE 5th International Symposium on, June 2013, pp. 1-5. 